\title{
Rou Cong Rong
}

National Cancer Institute

\section{Source}

National Cancer Institute. Rou Cong Rong. NCI Thesaurus. Code C129586.

A Chinese herbal medicine ( $\mathrm{CHM}$ ) derived from the stems of the herbaceous plant Cistanche deserticola Y. C. Ma (Ginseng of the desert) belonging to the Orobanchaceae family. Rou Cong Rong may have antioxidative and neuroprotective activities. This traditional Chinese medicine (TCM) is used to help with pain, deficiency of kidney yang and may relieve constipation by moistening and smoothing stool. 